\title{
Methods of multi-dimensional statistical analysis for assessing the impact of applying technology of paint formulation
}

\author{
Valentina Loganina ${ }^{{ }^{*}}$, and Elena Kuimova ${ }^{l}$ \\ ${ }^{1}$ Penza State University of Architecture and Construction, 440028, 28, Street Titov, Penza, Russia
}

\begin{abstract}
Application information of the method of canonical correlations to assess the influence of technological factors on the quality of paint coat of building products and structures are provided below. The algorithm for calculating the method of canonical correlations is considered. It is shown that the substrate porosity is determinative for the paint coat quality. The viscosity of the paint applied by the pneumatic method affects the quality indicators less, especially compare to brush application. The coefficients in canonical variables characterize the strength of the influence of the relevant signs-factors and the efficiency of indicators on the level of communication between them. Various ways of applying paint are considered on substrate with a porosity of $24 \%, 28 \%, 32 \%$ on the example of oil paints MA-15, of alkyd paint PF-115, of water dispersion paint AK111 , it is shown, that the porosity of the substrate is a decisive factor in determining the quality of the paint coating. The viscosity of the paint when applied by the pneumatic method affects the quality indicators less compared to the brush application.
\end{abstract}

\section{Introduction}

Previous studies show that the resistance of paint coat, among other factors, is determined by the quality of the external appearance of coat $[1,2]$. The quality of the appearance of the coatings is significantly influenced by the technology of applying paint, its rheological properties, and the quality of the painted surface. Meanwhile, the process of creating paint coatings on a porous cement substrate is often unstable and irreproducible $[3,4]$. In this connection, the assessment of the most significant factors influencing the quality of coating is relevant. Current knowledge of the possibilities of mathematical processing of statistical information and the interpretation of the results obtained allows us to investigate the interrelationships of individual sides of technological processes with the involvement of several effective factors in the analysis process. We consider the possibility of using method of multidimensional statistical analysis which are based on the hypothesis about the possibility of studying the existing relationships between the observed phenomena indirectly, consisting in constructing correlation matrices and recognizing them based on factor estimates to identify the causes of coating quality and defect

*Corresponding author:loganin@mail.ru 
Let us consider the possibility of using multidimensional statistical analysis methods for identify the causes of coating quality deterioration and the appearance of defects [5] . Quality is determined by many different quantities - quality indicators $y=\left(y_{1}, y_{2}, \ldots, y_{n}\right)$. There are also many technological factors $x=\left(x_{1}, x_{2}, \ldots, x_{m}\right)$. Between records of measured values of factors and indicators, there is a correspondence $y_{q} \leftrightarrow x_{q}, q=1,2, \ldots, Q$, where $\mathrm{Q}$ is the number of realizations. We try to determine the share contributed by individual technological factors to the overall instability of the process.

The method of canonical correlations make it possible to contemporary analyze the relationship between several output parameters and a large number of determining factors. It does not require a lack of correlation, both in the group of performance indicators and in the group of factor indicators.

The algorithm for calculating the method of canonical correlations is constructed in such a way that the original variables are replaced by their linear combinations, which are linearly independent. At the same time, there is a high degree of connection between linear combinations of factors and linear combinations of output parameters. The coefficients in canonical variables characterize the strength of the influence of the relevant signs-factors and the performance of indicators on the level of communication between them.

\section{Materials and research methods}

Briefly give the essence of the method. First of all, the covariance matrix is calculated from the array of measured factor values, which is a characteristic of the interaction and its changes:

$$
S=\left(\begin{array}{ccccccc}
K_{x_{1} x_{1}} & K_{x_{1} x_{2}} & \ldots & K_{x_{1} x_{m}} & K_{x_{1} y_{1}} & \ldots & K_{x_{1} y_{n}} \\
K_{x_{2} x_{1}} & K_{x_{2} x_{2}} & \ldots & K_{x_{2} x_{m}} & K_{x_{2} y_{1}} & \ldots & K_{x_{2} y_{n}} \\
\ldots & \ldots & \ldots & \ldots & \ldots & \ldots & \ldots \\
K_{x_{m} x_{1}} & K_{x_{m} x_{2}} & \ldots & K_{x_{m} x_{m}} & K_{x_{m} y_{1}} & \ldots & K_{x_{m} y_{n}} \\
K_{y_{1} x_{1}} & K_{y_{1} x_{2}} & \ldots & K_{y_{1} x_{m}} & K_{y_{1} y_{1}} & \ldots & K_{y_{1} y_{n}} \\
\ldots & \ldots & \ldots & \ldots & \ldots & \ldots & \ldots \\
K_{y_{n} x_{1}} & K_{y_{n} x_{2}} & \ldots & K_{y_{n} x_{m}} & K_{y_{n} y_{n}} & \ldots & K_{y_{n} y_{n}}
\end{array}\right)
$$

Where $K_{x_{i} x_{j}}=M\left(\left(x_{i}-\overline{x_{i}}\right) \cdot\left(x_{j}-\overline{x_{j}}\right)\right)$ is the covariance of the specified variables, then the matrix $\mathrm{S}$ is:

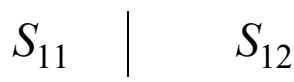




\section{$S_{21} \quad S_{22}$}

presented in the form of a block matrix. In fact, we divide the matrix $\mathrm{S}$ into four parts:

where $S_{11}$ is the covariance matrix of factors $x_{1}, x_{2}, \ldots, x_{m}$, the dimension of the matrix $(m \times m)$;

$S_{22}$ - covariance matrix of indicators $y_{1}, y_{2}, \ldots, y_{n}$, the dimension of the matrix $(n \times n)$;

$S_{12}$ - covariance matrix containing the covariance $K_{x_{i} y_{j}, i=1, m}, j=\overline{1, n}$, the dimension of the matrix $(m \times n)$.

$S_{21}$ - is the result of the matrix transposition $S_{12}$.

The canonical correlation coefficients can be calculated on the basis of the sample correlation matrix, especially if you have to work with data of incomparable units of measurement

\begin{tabular}{l|l}
$R_{11}$ & $R_{12}$ \\
\hline$R_{21}$ & $R_{22}$
\end{tabular}

The task of determining the maximum correlation between canonical variables $U=U_{1} x_{1}+U_{2} x_{2}+\ldots+U_{m} x_{m}$ and $V=V_{1} y_{1}+V_{2} y_{2}+\ldots+V_{n} y_{n} \quad$ reduced to the problem of determining the eigenvalues of matrices $R_{11}^{-1} R_{12} R_{22}^{-1} R_{12}^{T}$ and $R_{22}^{-1} R_{12}^{T} R_{11}^{-1} R_{12}$ and their eigenvectors. Given that the dimension of these matrices we obtain $n$ eigenvalues $\lambda_{1}^{2} \geq \lambda_{2}^{2} \geq \ldots \geq \lambda_{n}^{2}$, m eigenvectors of $\mathrm{U}$, and $\mathrm{n}$ eigenvectors of $\mathrm{V}$.

To analyze the influence of the method of applying the paint composition, its rheological properties and the porosity of the substrate on the surface quality of the coatings, we performed the following experiment. Colorful compositions with different rheological characteristics were applied to the mortar substrate with a porosity of $24 \%$, $28 \%, 32 \%$ in two layers with intermediate drying for 20 minutes. Before applying the paint, the substrate surface was primed. In addition, part of the mortar samples was leveled with spackling compounds. The rheological properties of paints were evaluated in terms of their conditional dynamic viscosity and surface tension. As the paint compositions, alkyd enamel of the PF-115 brand, oil paint of the MA-15 brand, acrylic water-dispersion (front) paint were used. Paint were applied by pneumatic method, brush. The surface quality of the coatings was evaluated by the roughness and adhesion strength of the coatings. The surface roughness of the coating was determined using a TR-100 profilograph device, and the adhesion strength was determined by the method of puck separation.

\section{Research result}


Analysis of the data (Table 1) shows that the value of the surface roughness of the coating depends on the method of applying the paint composition, its rheological properties and the porosity of the cement substrate. So, for MA15 oil paint (green color), the minimum roughness value equal to $\mathrm{Ra}=3.12 \mathrm{mkm}$ is achieved on a substrate with a porosity of $\mathrm{P}=$ $24 \%$ with a paint viscosity of $0.00261 .103 \mathrm{~Pa}$.s when applied with a brush.

For paint PF-115, the minimum roughness value equal to $\mathrm{Ra}=1.3 \mathrm{mkm}$ is achieved on a substrate with a porosity of $\mathrm{P}=28 \%$ with a paint viscosity of $0.00065 .103 \mathrm{~Pa}$.s when applied with a brush. For water dispersion paint, a minimum roughness value equal to $\mathrm{Ra}=$ $3.5 \mathrm{mkm}$ is achieved on a substrate with a porosity of $\mathrm{P}=32 \%$ with a paint viscosity of $0.013 .103 \mathrm{~Pa}$. with a porosity of $\mathrm{P}=24 \%$ with a viscosity of paint $0.0347 .103 \mathrm{~Pa}$. s when applied with a brush. The minimum roughness value is characteristic of the surface of the coatings on the putty substrate, regardless of the method of application and the rheological properties of the paint formulations.

Table 1. Surface roughness of coatings.

\begin{tabular}{|c|c|c|c|c|c|}
\hline \multirow[t]{4}{*}{ Paint type } & \multirow{4}{*}{$\begin{array}{l}\text { Substrate } \\
\text { porosity, \% }\end{array}$} & \multicolumn{4}{|c|}{ Roughness, Ra, mkm } \\
\hline & & \multicolumn{4}{|c|}{ Application Method } \\
\hline & & \multicolumn{2}{|c|}{ Brush } & \multicolumn{2}{|c|}{ Pneumatic method } \\
\hline & & $\eta_{1}$ & $\eta_{2}$ & $\eta_{1}$ & $\eta_{2}$ \\
\hline \multirow[t]{4}{*}{ PF-115 } & 24 & 3,14 & 3,26 & 6,7 & 4,7 \\
\hline & 28 & 1,78 & 1,3 & 7,65 & 7,47 \\
\hline & 32 & 4,34 & 2,57 & 6,98 & 5,31 \\
\hline & 0 (putty) & 1,28 & 1,8 & 2,6 & 1,18 \\
\hline \multirow{4}{*}{ MA-15 } & 24 & 3,12 & 7,4 & 4,37 & 5,27 \\
\hline & 28 & 4,3 & 6,27 & 4,53 & 9,07 \\
\hline & 32 & 5,65 & 3,76 & 5,4 & 6,42 \\
\hline & 0 (putty) & 1,79 & 2,54 & 2,8 & 3,2 \\
\hline \multirow{4}{*}{$\begin{array}{c}\text { Water } \\
\text { dispersion }\end{array}$} & 24 & 6,5 & 4,7 & - & - \\
\hline & 28 & 4,8 & 3,3 & - & - \\
\hline & 32 & 3,5 & 3,7 & - & - \\
\hline & 0 (putty.) & 2,57 & 3,08 & - & - \\
\hline
\end{tabular}

Note: for paint PF-115 $\eta 1=0.001 * 103$ Pa.s, $\eta 2=0.00065 * 103$ Pa.s ,; for MA-115 paint $\eta 1=0.0026 * 103$ Pa.s, $\eta 2=0.00210 * 103$ Pa.s ,; for water dispersion paint $\eta 1=0.0347 * 103$ Pa.s, $\eta 2=0.02317 * 103$ Pa.s,

Below is an example of the stages of processing the initial information

Table 2. Properties of coatings based on MA-15 paint 


\begin{tabular}{|c|c|c|c|c|}
\hline Paint type & $\begin{array}{c}\text { Substrate } \\
\text { poro-sity, } \%, x_{1}\end{array}$ & $\begin{array}{c}\text { Paint } \\
\text { viscosity } \\
\eta \cdot 10^{3} \text {, Пa.c } \\
x_{2} \\
\end{array}$ & $\begin{array}{c}\text { Roughness } \\
\text { coating, } \mathrm{mkm}, y_{1}\end{array}$ & $\begin{array}{c}\text { Adhesion strength, MPa } \\
y_{2}\end{array}$ \\
\hline \multirow{8}{*}{$\begin{array}{c}\text { MA-15 } \\
\text { brush }\end{array}$} & 24 & 0,0026 & 3,12 & 1,4 \\
\hline & 24 & 0,0021 & 7,4 & 1,1 \\
\hline & 28 & 0,0026 & 4,3 & 1,2 \\
\hline & 28 & 0,0021 & 6,27 & 0,7 \\
\hline & 32 & 0,0026 & 5,65 & 0,6 \\
\hline & 32 & 0,0021 & 3,76 & 0,9 \\
\hline & 0 & 0,0026 & 1,79 & 1,8 \\
\hline & 0 & 0,0021 & 2,54 & 1,7 \\
\hline
\end{tabular}

Since the variances of the factor variables differ significantly from one another and there are disparate units of measurement, it is reasonable to use a correlation matrix (Table 3).

Table 3. The effect of substrate porosity on the adhesion strength of coatings

\begin{tabular}{|c|c|c|c|c|}
\hline Indicators & $\begin{array}{c}\text { Substrate } \\
\text { porosity } \boldsymbol{x}_{\boldsymbol{1}}\end{array}$ & $\begin{array}{c}\text { Paint } \\
\text { viscosity } \boldsymbol{x}_{\mathbf{2}}\end{array}$ & Roughness $\boldsymbol{y}_{\boldsymbol{1}}$ & $\begin{array}{c}\text { Adhesion } \\
\text { strength } \boldsymbol{y}_{\mathbf{2}}\end{array}$ \\
\hline porosity $x_{1}$ & 1 & 0 & 0,6532 & $-0,8831$ \\
\hline viscosity $x_{2}$ & 0 & 1 & $-0,3518$ & 0,1822 \\
\hline Roughness $y_{1}$ & 0,6532 & $-0,3519$ & 1 & $-0,7619$ \\
\hline Adhesion strength $y_{2}$ & $-0,8831$ & 0,1822 & $-0,7619$ & 1 \\
\hline
\end{tabular}

Thus, further work with the matrix

$$
R=\left(\begin{array}{cccc}
1 & 0 & 0,6532 & -0,8831 \\
0 & 1 & -0,3518 & 0,1822 \\
0,6532 & -0,3518 & 1 & -0,7619 \\
-0,8831 & 0,1822 & -0,7619 & 1
\end{array}\right)
$$

Correlation matrix of factor variables $x_{1}$ and $x_{2}$ :

$$
R_{11}=\left(\begin{array}{ll}
1 & 0 \\
0 & 1
\end{array}\right)
$$

Correlation matrix of quality indicators $y_{1 \text { and }} y_{2}$

$$
R_{22}=\left(\begin{array}{cc}
1 & -0,7619 \\
-0,7619 & 1
\end{array}\right)
$$


fficients of variables $x_{1}, x_{2}, y_{1}, y_{2}$. Wherein $R_{21}=R_{12}^{T}$

$$
R_{12}=\left(\begin{array}{cc}
0,6532 & -0,8831 \\
-0,3518 & 0,1822
\end{array}\right)_{\text {contains pairwise coupling }}
$$

Stage 3. Construction and calculation of the eigenvalues of the auxiliary matrix

Auxiliary matrix has the form:

$$
C=R_{11}^{-1} R_{12} R_{22}^{-1} R_{12}^{T}=\left(\begin{array}{cc}
0,7808 & -0,1510 \\
-0,1510 & 0,1413
\end{array}\right)
$$

The solution of the equation gives the following results on the eigenvalues of the matrix C: $\lambda_{1}^{2}=0,815, \lambda_{2}^{2}=0,107$. Corresponding eigenvectors

$$
\begin{aligned}
& \lambda_{1}{ }^{2}=0,815, \overrightarrow{\theta_{1}}=(0,976 ;-0,219) \\
& \lambda_{2}{ }^{2}=0,107 \overrightarrow{\theta_{2}}=(0,219 ; 0,976)
\end{aligned}
$$

The maximum eigen value $\lambda_{1}=0,903$, which indicates a very close relationship of the considered factors and quality indicators.

We obtain the canonical combination of technological factors: $0,976 x_{1}-0,219 x_{2}$. Thus, the contribution of the first factor variable to the overall instability of the quality indicators is more than 4 times greater than the contribution of the second factor.

When changing the method of applying paint on a pneumatic, canonical combination for coatings based on MA-15 paint is as follows: $0,999 x_{1}+0,054 x_{2}, \lambda_{1}=0,766$

Studying the main canonical correlations for coatings based on MA-15 paint with different methods of its application, it can be noted that in both linear combinations the greatest coefficient is preserved (substrate porosity). From this we can conclude that it is the porosity of the substrate that in this context is the decisive factor determining the quality of the paint coating. The viscosity of the paint (factor) when applied by the pneumatic method affects the quality indicators by an order of magnitude weaker compared to the brush application. Similar conclusions can be made on other studied paints (Table 4).

Table 4. Canonical correlations for coatings with different paint application methods

\begin{tabular}{|c|c|c|}
\hline PF-115 brush & $0,993 x_{1}-0,115 x_{2}$ & $\lambda_{1}=0,678$ \\
\hline PF-115 pneumatic & $0,932 x_{1}-0,363 x_{2}$ & $\lambda_{1}=0,921$ \\
\hline WD brush & $0,995 x_{1}+0,1 x_{2}$ & $\lambda_{1}=0,89$ \\
\hline
\end{tabular}

\section{Conclusions}

It should be added that the methods of multivariate analysis for any set of factors should be considered as a more complex stage of analysis. Its distinctive feature is not only the classification of factors for major and minor, but the rotation of factors in a spiral, tracking their influence on each other and their influence on performance indicators many times, in order to assess the various trends in their interaction. So, for example, it is of undoubted interest to replace by the calculating the covariance or correlation matrix, the average characteristics of the factors by their preferred in terms of quality, or provided for by 
regulatory documents. The study of the dynamics of the factor characteristics of the instability of the result can be an important component of detecting and eliminating the causes of poor-quality coating.

\section{References}

1. V.I.Loganina, G.A. Fokin, N.N Laskov, J. Eng. App. Sci., 2411-2413 (2016)

2. V.I.Loganina, Yu.P. Skachkov, V.S. Demyanova, Int. J. Eng. Tech., 7(4), 4651-4653 (2018)

3. V.I.Loganina, Yu.P. Skachkov, Assessment of the Stress State of the Coating in Depending on the Porosity of the Cement Substrate, Key Engineering Materials, Volume: Engineering Materials and Technology 737,179-183 (2016)

4. V.I.Loganina, L.V.Makarova, R.V. Tarasov, Ca. Stu. Const. Mate., 4, 81-84 (2016)

5. L.Albayrak, K. Khanipov, G. Golovko, Detection of multi-dimensional co-exclusion patterns in microbial communities. Bioinformatics, 3695-3701 (2018) 